\title{
Optical Diagnostics of Shock-induced Argon Plasmas Based on a Simple Collisional-Radiative Model*
}

\author{
By Shin TAKeUCHI, ${ }^{1)}$ Gouji YAMADA, ${ }^{2)}$ Chihiro TAKAHASH ${ }^{2)}$ and Hiromitsu KaWAZOE ${ }^{1)}$ \\ ${ }^{1)}$ Department of Mechanical and Aerospace Engineering, Tottori University, Tottori, Japan \\ ${ }^{2)}$ Department of Mechanical Engineering, Tokai University, Hiratsuka, Japan
}

(Received March 12th, 2017)

\begin{abstract}
In the present study, the optical diagnostics of nonequilibrium argon plasma induced by a hypersonic shock wave are conducted based on a simple collisional-radiative (CR) model. Spectroscopic measurements show that line spectra of argon atoms are predominant and clearly seen in the near-infrared region. Line spectra of argon atoms and ion are observed in the visible region. However, the influence of contaminated gases is significant. The electron excitation population is deduced by applying the Boltzmann plot method to the measured line spectra of argon atom. The result shows that the electron excitation population is much different from the Boltzmann distribution, indicating that the argon plasma is in a thermal nonequilibrium state. Finally, the spatial distribution of the electron temperature is obtained by applying the CR model to the measured spectra and compared with that of the calculated one. It is found that the electron temperature obtained using the CR model qualitatively agrees well with the calculated one. The present result has indicated the possibility of using the simple CR model for determining the electron temperature of nonequilibrium argon plasma induced by a hypersonic shock wave.
\end{abstract}

Key Words: Shock Wave, Electron Temperature, CR Model, Argon Plasma, Shock Tube

\section{Introduction}

Characteristics of nonequilibrium plasmas around space vehicles entering into a planetary atmosphere should be clarified for the accurate prediction of flight environments. Experimental and numerical studies have been carried out around the world to characterize nonequilibrium plasmas in entry flight conditions. As a result, some physical models for numerical analysis have been developed and used to predict flight environments. ${ }^{1-4)}$ The validation of the models has been carried out for many years based on shock tube experiments to improve prediction accuracy. One of the authors conducted shock-layer radiation measurements in $\mathrm{N}_{2}$ using a shock tube.,5) The results were compared with the numerical prediction of the two-temperature mod$\mathrm{el}^{4)}$ proposed by Park, showing that a discrepancy of the model for the relaxation process of internal states behind a shock wave. In addition, numerical analysis implied that precursor electrons are generated ahead of the shock wave and have a great influence on the relaxation process behind the shock wave. ${ }^{7}$ To understand the thermochemical nonequilibrium processes behind a shock wave, shock tube experiments were conducted using argon as the test gas because argon atoms have fewer energy modes than molecules, thereby simplifying the phenomena. Triple probe measurements showed that precursor electrons and argon ions were mainly generated by photoionization, making use

(C) 2017 The Japan Society for Aeronautical and Space Sciences

*Presented at the 2016 Asia-Pacific International Symposium on Aerospace Technology (APISAT-2016), October 25-27, 2016, Toyama, Japan of the radiation energy behind a shock wave. ${ }^{8)}$ In addition, spectroscopic measurements showed that the electron density behind the shock wave tended to drop due to the radiative energy loss from the test gas. ${ }^{9)}$ The energy loss is considered to be the energy source of the photoionization. These results pointed out that the thermochemical state around the shock wave was out of equilibrium. Therefore, a conventional method based on local thermodynamic equilibrium (LTE) is not applicable to the argon plasma in the present condition. In this study, we newly apply a collisional-radiative (CR) model to nonequilibrium argon plasma induced by a hypersonic shock wave to investigate the ionization process. CR models deal with electronic transitions between all energy levels by taking elementary processes into account. They are useful tool to diagnose nonequilibrium plasmas. Several CR models for argon have been developed in past studies. ${ }^{10-14)}$ The models consider different argon energy levels and elementary processes. In these models, the analytical forms proposed by Drawin are particularly well adapted for argon plasmas to deduce rate coefficients of electronic transitions between excited levels. However, compared with the electron-impact processes from the ground state, cross-section data available for electron impact transitions between excited levels are very scarce. This is a drawback to applying the CR model to plasma diagnostics. In past studies, a simple CR model was proposed for argon plasma and the validity was examined by applying it to an argon plasma in a positive column of dc discharge. ${ }^{15)}$ In this model, electronic transitions are considered for a certain energy level instead of all energy levels, simplifying the atomic processes in the CR model. The argon plasma treated in the study is an ion- 
izing plasma where the nonequilibrium phenomenon is relatively weak because the gas pressure is on the order of a few thousand $\mathrm{Pa}$ and the flow velocity is subsonic. On the other hand, we focus on argon plasma, where the nonequilibrium phenomenon is strong because the gas pressure is a few tens of $\mathrm{Pa}$ and the flow velocity is hypersonic. The validity of the simple CR model has not been investigated for a nonequilibrium argon plasma. So, in this study, we newly apply the simple CR model to a nonequilibrium argon plasma to expand the range of the applicability. The purpose of this study is to investigate the characteristics of a nonequilibrium argon plasma induced by a hypersonic shock wave based on the CR model.

\section{Experimental Setup}

\subsection{Shock tube facility}

A free piston-driven shock tube is used in this study to generate argon plasmas induced by a hypersonic shock tube. The shock tube facility consists of a high-pressure reservoir, compression tube, low pressure tube containing the test section, and vacuum tank. ${ }^{16)}$ The total length of the shock tube is about $7 \mathrm{~m}$. The test section, with a $44 \mathrm{~mm}$ square cross-section, is made of aluminum alloy to reduce the influence of emission from contaminated gases in the test gas. Four quartz windows with a diameter of $30 \mathrm{~mm}$ are equipped with the test section wall. The test section can be evacuated to a pressure of $0.3 \mathrm{~Pa}$ using a roots pump (Edwards, QMB500) backed up by an oil rotary pump (Edwards, E2M80). A previous study showed that the shock tube cac simulate the typical entry flight conditions for Earth entry and Mars entry.

\subsection{Optical measurement system}

Figure 1 shows a schematic of the optical measurement system at the test section. The shock velocity at the test section is measured using the double-laser schlieren measurement system. Two laser beams are placed along the flow direction to pass through the test section perpendicularly to the axis of the shock-tube flow. The optical path is adjusted by flat mirrors to reach separate avalanche photodiodes (APD). The laser beams deflect due to the density gradient at the shock front, causing a change in the output signals of APD. The shock velocity at the test section can be obtained from the beam distance and time difference of the APD output signals.

Shock layer radiation measurements are conducted using a multipoint spectroscopic measurement system with a short test duration. The measurement system is composed of a fiber alley, convex lens, imaging spectrometer, and ICCD camera. The fiber alley has ten fiber elements equally aligned at $1.0 \mathrm{~mm}$ intervals on the light-collecting surface parallel to the axis of the shock-tube flow. The convex lens is aligned to focus the image of the axis of the shock-tube flow at the same magnification. The measurement system enables us to obtain ten multipoint spectra at $1.0 \mathrm{~mm}$ intervals along the flow direction at a time. Figure 2 shows an example of the multipoint spectrum image obtained using the measurement system. The spatial components of 40 pixels are accumulated for each spectrum to

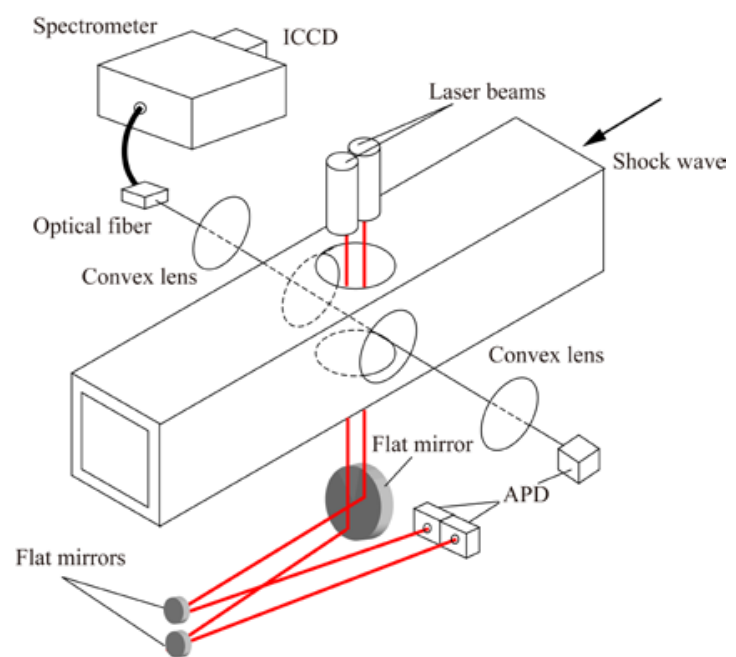

Fig. 1. Optical measurement system.

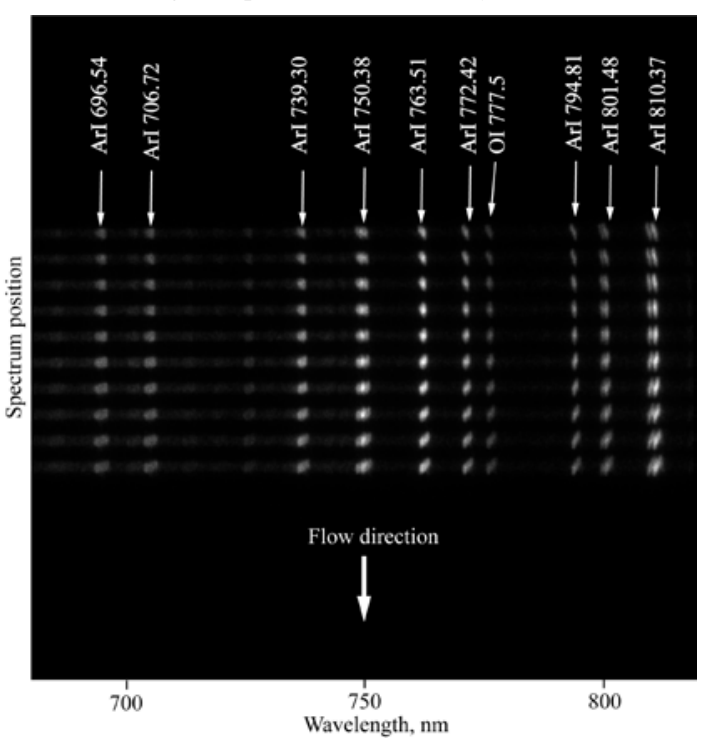

Fig. 2. Multipoint spectrum image.

increase the signal-to-noise ratio and multipoint spectra are extracted from the spectrum image. The spectroscopic measurement system is calibrated using a standard light source (Newport, 63355) to deduce the relative intensity from the measured intensity. In addition, the measurement system is calibrated using a mercury argon calibration source (Ocean Optics, HG-1) to improve the wavelength accuracy.

The total radiation intensity is measured using an APD placed at the other side of the spectrometer, and the signal is used to trigger the ICCD camera at the instant the shock arrives.

\subsection{Test conditions}

Test conditions are shown in Table 1 . In this study, argon is used as the test gas. Nominal shock velocity is $5.81 \pm 0.13 \mathrm{~km} / \mathrm{s}$ and the test gas pressure is $50 \mathrm{~Pa}$. The spectrometer is operated with a grating of 300 lines $/ \mathrm{mm}$ and slit width of $300 \mu \mathrm{m}$, providing a wavelength resolution of $0.14 \mathrm{~nm} /$ pixel. The ICCD camera is triggered using an APD output signal with a gate time of 50 ns. The trigger timing is controlled by the on-board delay pulse generator of the ICCD camera. 
Table 1. Test conditions.

\begin{tabular}{|c|c|}
\hline Test gas & Argon \\
\hline Shock velocity, $\mathrm{km} / \mathrm{s}$ & $5.81 \pm 0.13$ \\
\hline Test gas pressure, $\mathrm{Pa}$ & 50 \\
\hline Grating, lines/mm & 300 \\
\hline Slit width, $\mu \mathrm{m}$ & 300 \\
\hline Gate time of ICCD, ns & 50 \\
\hline
\end{tabular}

\section{Theory of CR Model}

\subsection{A basic CR model for the argon atom}

There are various types of CR models for the argon atom. The models consider different energy levels and elementary processes. We apply the CR model developed by VIček. ${ }^{11)}$ In this model, electronic transitions due to collisional and radiative processes are considered for 65 effective energy levels. The rate equation for the $p$ th level is given by

$$
\begin{aligned}
\frac{d}{d t} N_{p} & =\sum_{q<p} C_{q, p} N_{e} N_{q}+\sum_{q>p}\left[F_{q, p} N_{e}+A_{q, p}\right] N_{q} \\
& +\left[\beta_{p}+\alpha_{p} N_{e}\right] N_{e} N_{i} \\
& -\left[\sum_{q>p} C_{p, q} N_{e}+\sum_{q<p} F_{p, q} N_{e}+\sum_{q<p} A_{p, q}+S_{p} N_{e}\right] N_{p}
\end{aligned}
$$

where $C_{p, q}$ is the rate coefficient for electron impact excitation from the $p$ th to $q$ th level. $F_{p, q}$ is the rate coefficient for electron impact de-excitation from $p$ th to $q$ th level. $S_{p}$ is the rate coefficient for electron impact ionization. $A_{p, q}$ is the transition probability from the $p$ th to $q$ th level. $\alpha_{p}$ is the rate coefficient for inverse three body recombination. $\beta_{p}$ is the radiative recombination rate coefficient. $N_{p}$ is the population density of the $p$ th level. $N_{e}$ and $N_{i}$ are the electron density and ion density, respectively. Since the relaxation time for excited states is much shorter than that for the ground state, the following relation can be assumed, except the ground state.

$$
\frac{d}{d t} N_{p}=0 \quad(p=2,3,4 \ldots \ldots 65)
$$

Thus, Eq. (1) can be described as

$$
\begin{aligned}
& \sum_{q<p} C_{q, p} N_{e} N_{q}+\sum_{q>p}\left[F_{q, p} N_{e}+A_{q, p}\right] N_{q} \\
& +\left[\beta_{p}+\alpha_{p} N_{e}\right] N_{e} N_{i} \\
& -\left[\sum_{q>p} C_{p, q} N_{e}+\sum_{q<p} F_{p, q} N_{e}+\sum_{q<p} A_{p, q}+S_{p} N_{e}\right] N_{p}=0
\end{aligned}
$$

By applying Eq. (3) to all excited levels except the ground state, 64 simultaneous equations are obtained. In these equations, unknown parameters are the electron temperature, electron density, and populations of excited levels. A solution of the simultaneous equations with the input parameters of electron temperature and electron density gives populations of excited levels. On the contrary, if populations of excited levels are known quantities, the electron temperature and electron density can be obtained from the simultaneous equations.

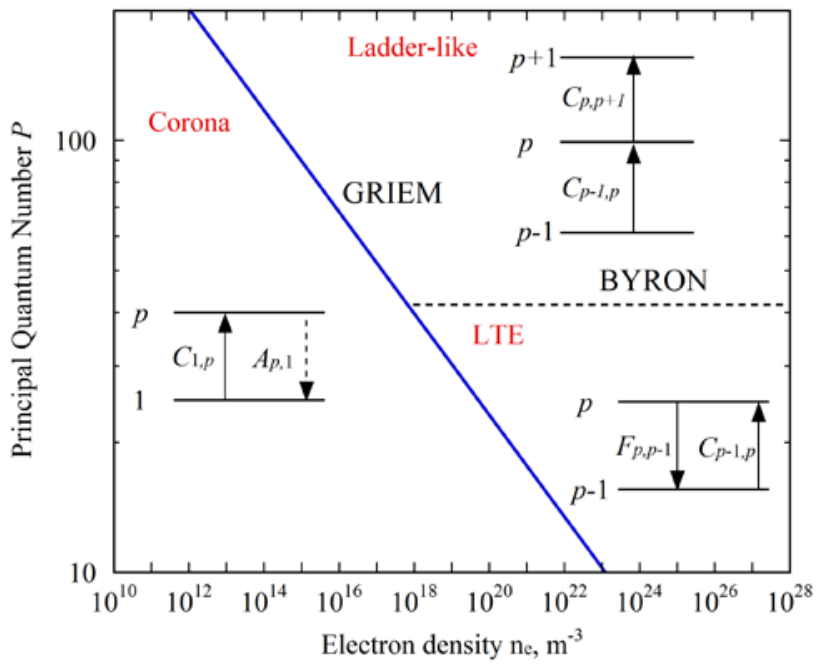

Fig.3. Phase diagram of ionizing plasma for argon atom.

\subsection{Principle}

In the present study, we focus on the argon plasma induced by a hypersonic shock tube. Argon is excited and ionized into plasma due to the high pressure and temperature generated by a hypersonic shock wave. The plasma is considered to be an ionizing plasma. Figure 3 shows the phase diagram of ionizing plasma for argon atoms. In this figure, the blue line shows Griem's boundary described by the following equation ${ }^{17)}$

$$
p_{G} \cong 480\left(N_{e} / z^{7}\right)^{-2 / 17}
$$

where $z$ is the nuclear charge. On the other hand, the dashed line shows Byron's boundary at $T_{e}=1 \mathrm{eV}$ described by the following equation

$$
P_{B} \cong\left(z^{2} R / 3 k_{B} T_{e}\right)^{1 / 2}
$$

where $R, k_{\mathrm{B}}$, and $T_{\mathrm{e}}$ are Rydberg constant, Boltzmann constant and electron temperature, respectively. For atoms and ions except hydrogen-like ions, the effective principle quantum number given by the following equation is used

$$
P^{*}=z\left[R / \chi_{i}\right]
$$

where $\chi_{i}$ is the ionization energy of the $i$ state. In the low electron density regime under Griem's boundary, plasma is assumed to be in corona equilibrium where the inflow populations resulting from electron impact excitation equals the outflow population as the result of radiative transition between the excited and ground states. In the high electron density regime over Griem's boundary, there are two phases divided by Byron's boundary. In the phase under Byron's boundary, local thermal equilibrium (LTE) is satisfied, where electron impact excitation and de-excitation processes are balanced. In the phase over the Byron's boundary, ladder-like electron impact transition is dominant. Our past study shows that the electron density of argon plasma induced by a hypersonic shock tube is on the order of $10^{21} \mathrm{~m}^{-3} .{ }^{9)}$ In addition, the effective principal quantum number for most energy levels is higher than Byron's boundary. Therefore, ladder-like electron impact transition is considered to be important for argon atoms in 
this condition. Here, let us consider the equilibrium of the ladder-like electron impact transition of level $p$ shown in Fig. 3. The steady-state rate equation is shown as follows.

$$
C_{p-1, p} N_{p-1}-C_{p, p+1} N_{p}=0
$$

where $C_{p-1, p}$ and $C_{p, p+1}$ are the rate coefficients for $p$ - 1 to $p$ and $p$ to $p+1$ electron impact excitations, respectively. $N_{p-1}$, $N_{p}$ are the population densities of the $p$-1th and the $p$ th level, respectively. The rate coefficients $C_{p-1, p}$ and $C_{p, p+1}$ are functions of the electron temperature, the solution of Eq. (4) with measured $N_{p-1}$ and $N_{p}$ enables us to obtain the electron temperature. The actual electronic transition processes are much more complex than that shown here. However, the simple CR model in this study is based on the idea of determining the electron temperature.

\subsection{A simple CR model for argon}

In the present study, the electron temperature is obtained from the measured spectrum using the simple CR model for argon. The basic idea of the CR model was originally developed by Kano et al. ${ }^{15)}$ and applied to the positive column of an argon dc discharge. In the model, electronic transitions for a certain energy level are considered to obtain the electron temperature. Figure 4 shows energy levels and the electronic transitions considered in this study. The electronic transition for the energy level $i=20$ is considered because the population densities for related energy levels are obtained using spectroscopic measurements in the visible region. The balance equation of the level $i=20$ is shown as follows.

$$
F_{25,20} N_{25}+F_{27,20} N_{27}=\left(C_{20.25}+C_{20,27}\right) N_{20}
$$

In the equation, population densities $N_{20}, N_{25}$, and $N_{27}$ are obtained by spectroscopic measurements for wavelengths of $739.3 \mathrm{~nm}, 383.5 \mathrm{~nm}$, and $682.7 \mathrm{~nm}$, respectively. The spectroscopic data are summarized in Table 2 . The solution for the equation using measured population densities gives the electron temperature of argon plasma. The rate coefficients $C_{i, j}$ for electron impact excitation is given by

$$
C_{i, j}=\sqrt{\frac{8 k_{B} T_{e}}{\pi m_{e}}} \int_{u_{i, j}}^{\infty} \sigma_{i, j} \cdot \exp (-u) \cdot u \cdot d u
$$

where $m_{\mathrm{e}}$ is the mass of the electron. $u_{i, j}$ is the dimensionless energy expressed as $u_{i, j}=\varepsilon_{i, j} /\left(k_{B} T_{e}\right)$ using the electron energy $\varepsilon_{i, j} \cdot \sigma_{i, j}$ is the cross-section for electron impact excitation. The corresponding rate coefficient $F_{j, i}$ for electron impact de-excitation is derived from detailed balance and is given by

$$
F_{j, i}=C_{i, j} \cdot \frac{g_{i}}{g_{j}} \cdot \exp \left(-u_{i, j}\right)
$$

The cross-section $\sigma_{i, j}$ is calculated using Drawin's formulae in this study. The cross-sections for allowed and forbidden transitions, $\sigma_{i, j}{ }^{A}, \sigma_{i, j}{ }^{F}$, are given as follows, respectively.

$$
\begin{aligned}
\sigma_{i, j}^{A} & =4 \pi a_{0}^{2}\left(\frac{\varepsilon_{1}^{H}}{\varepsilon_{i, j}}\right)^{2} f_{i, j} \alpha_{i, j}^{A} U_{i, j}^{-2}\left(U_{i, j}-1\right) \\
& \times \ln \left(1.25 \beta_{i, j} U_{i . j}\right)
\end{aligned}
$$

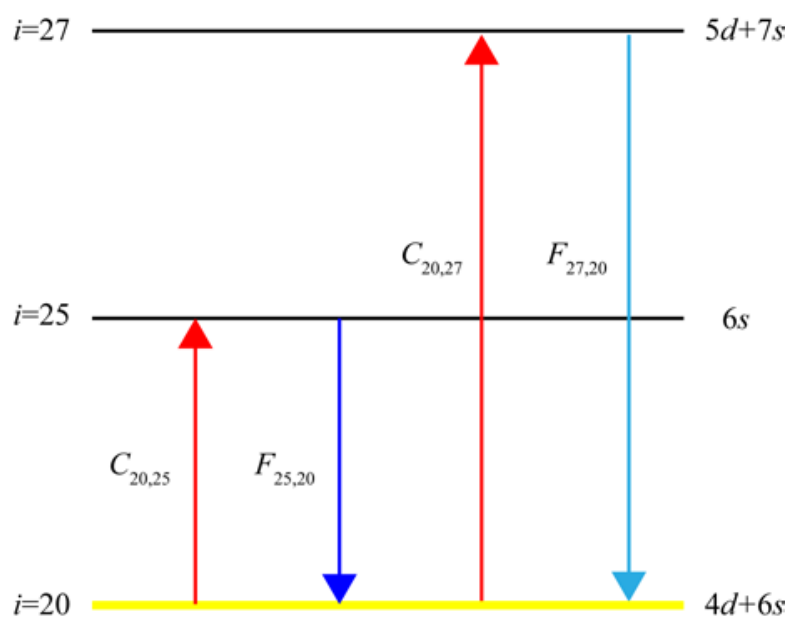

Fig.4. Electronic transition for a simplified CR model.

Table 2. Energy levels used in the CR model.

\begin{tabular}{|c|c|c|c|c|c|}
\hline$i$ & Designation & Transition & Wavelength & $\varepsilon_{\text {I }}$, & $g_{i}$ \\
\hline 20 & $4 d+6 s$ & $6 s[3 / 2]_{1}^{o} \rightarrow 4 p[3 / 2]_{2}$ & 739.3 & 14.792 & 3 \\
\hline 25 & $6 p$ & $6 p[1 / 2]_{0} \rightarrow 4 s^{\prime}[1 / 2]_{1}^{o}$ & 383.5 & 15.068 & 1 \\
\hline 27 & $5 d+7 s$ & $5 d[1 / 2]_{1}^{o} \rightarrow 4 p^{\prime}[3 / 2]_{2}$ & 682.7 & 15.118 & 3 \\
\hline
\end{tabular}

Table 3. Transition-dependent parameters used in the CR model.

\begin{tabular}{|c|c|c|c|c|c|c|c|c|}
\hline$i$ & $j$ & $\varepsilon_{i}$ & $\varepsilon_{j}$ & $g_{i}$ & $g_{j}$ & $f_{i, j} \alpha_{\mathrm{I}, j}{ }^{A}$ & $\beta_{I, j}$ & $\alpha_{\mathrm{I}, j}{ }^{P}$ \\
\hline 20 & 25 & 14.792 & 15.028 & 48 & 24 & $5.1 \times 10^{-1}$ & 1.0 & - \\
\hline 20 & 27 & 14.792 & 15.153 & 48 & 48 & - & - & $2.68 \times 10^{3}$ \\
\hline
\end{tabular}

$$
\sigma_{i, j}^{F}=4 \pi a_{0}^{2} \alpha_{i, j}^{P} U_{i, j}^{-1}\left(1-U_{i, j}^{-1}\right)
$$

where $a_{0}$ is the first Bohr radius of an atomic hydrogen. $f_{i, j}$ is the oscillator strength for electric dipole transition, an $U_{i, j}=\varepsilon / \varepsilon_{i, j} . \varepsilon_{1}{ }^{H}$ is the ionization energy for atomic hydrogen in the ground state. $f_{i, j} \alpha_{i, j}{ }^{A}, \beta_{i, j}$ and $\alpha_{i, j}{ }^{P}$ are transition dependent parameters and shown in Table 3. $A$ and $P$ symbolize the allowed and parity forbidden transitions, respectively.

\section{Results and Discussion}

\subsection{Measured spectrum}

Figures 5 (a) and 5 (b) show emission spectra obtained behind a shock wave in the visible (VIS) and near-infrared (NIR) regions, respectively. In Fig. 5 (a), the line spectra of argon atoms and ions are observed in the wavelength range from 400 to $430 \mathrm{~nm}$. These lines are closely packed and cannot be separated with the present wavelength resolution. CN Violet $\Delta \mathrm{v}=0$ and $\Delta \mathrm{v}=-1$ are observed. This is due to the impurity contained in the test gas. The $\mathrm{H}-\beta$ line of hydrogen is observed at $486 \mathrm{~nm}$. This is considered to come from the water vapor in the test section. The effect of contaminated gases is considered to be significant in the VIS region. In Fig. 5 (b), line spectra of the argon atoms are predominant and clearly separated from each other. A line spectrum of oxygen atoms is seen at $777 \mathrm{~nm}$, coming from the impurity in the test gas. No molecular spectra are seen in the spectrum. The effect of 


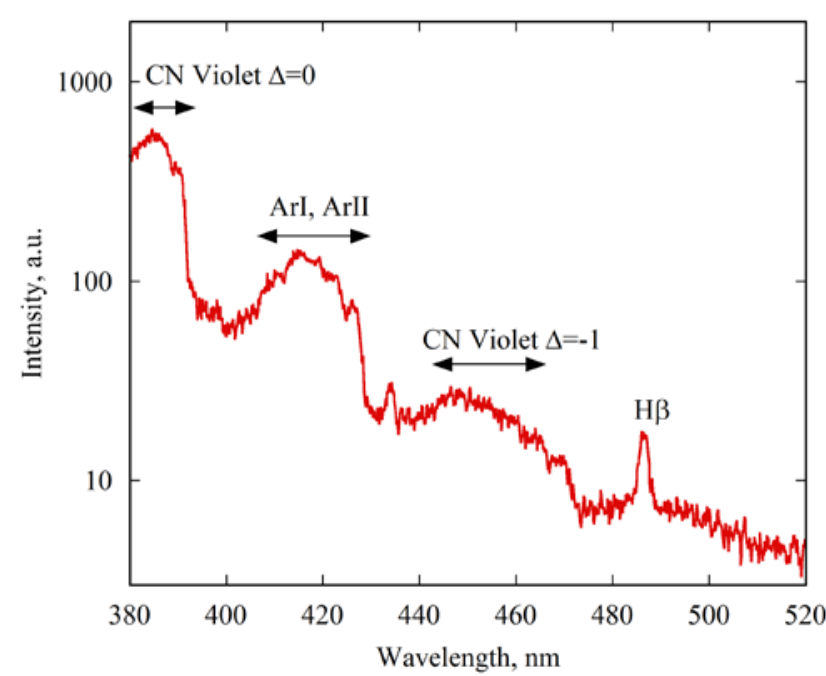

(a) In the VIS region

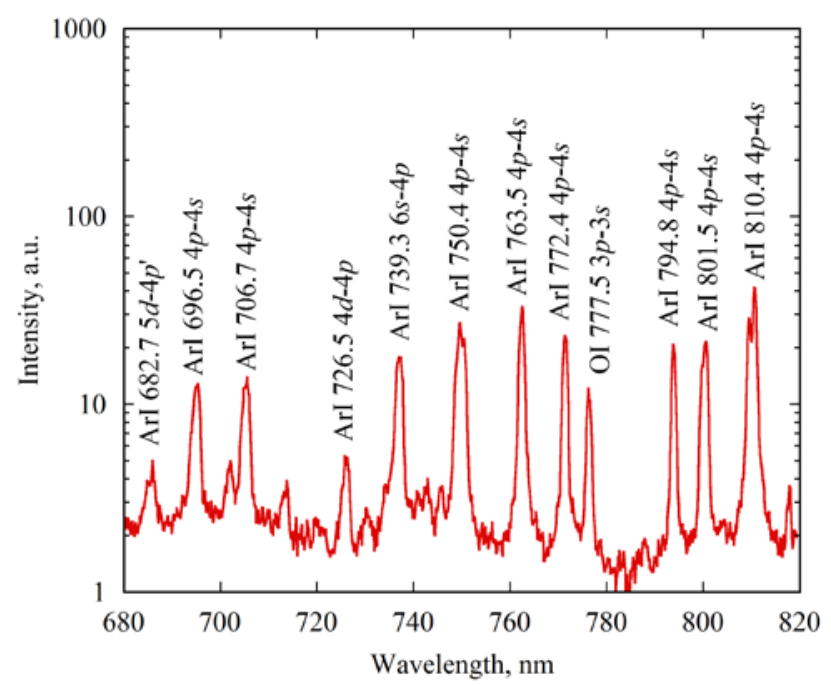

(b) In the NIR region

Fig. 5. Measured spectra.

contaminated gases is considered to be small in the NIR region.

The population densities, $N_{20}, N_{25}$, and $N_{27}$ should be obtained from the measured line spectra of the argon atom to substitute them into the Eq. (8). However, the population density $N_{25}$ cannot be obtained because the line spectrum of the argon atom at $383.5 \mathrm{~nm}$ is not clearly visible due to the influence of the $\mathrm{CN}$ Violet $\Delta \mathrm{v}=0$. In the ladder-like transition regime, the population distribution $N_{i} / g_{i}$ is proportional to the minus fifth power of the effective principle quantum number as shown below. ${ }^{17}$

$$
\frac{N_{i}}{g_{i}} \propto P^{*-5}
$$

$N_{25}$ is estimated by an interpolation of the population distribution applying the above relation for $N_{20}$ and $N_{27}$.

\subsection{Thermochemical state of argon plasma}

To investigate the thermochemical state of argon plasma behind the shock wave, the electronic excitation population is deduced using the Boltzmann plot method. In the method, it is assumed that the radiative gas is optically thin and the

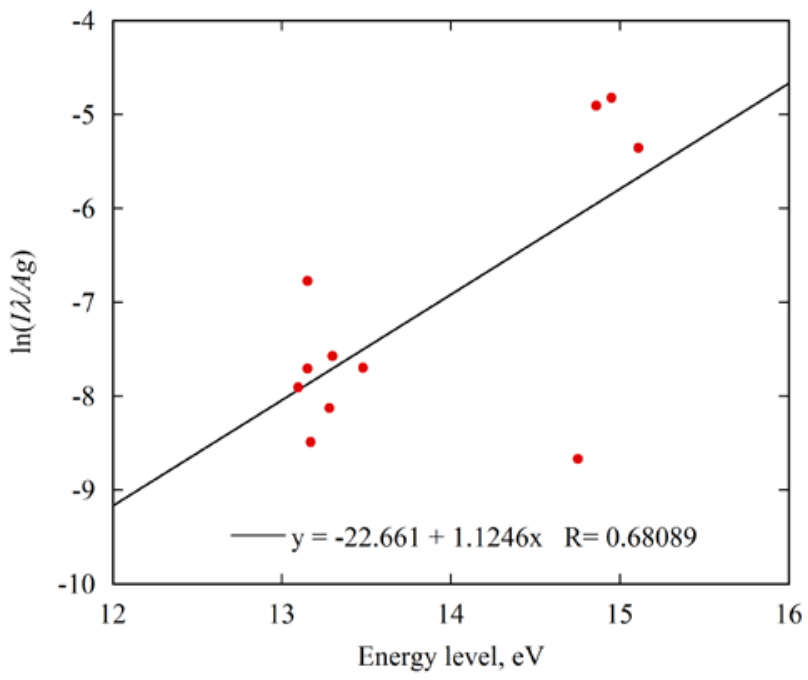

Fig. 6. Electronic excitation population.

absorption process can be neglected along the line of sight. In the assumption, the relative radiation intensity of an $i-j$ transition is considered to be proportional to the number density of the upper state $i$. The relation between the radiation intensity $I_{i, j}$ and the number density of the upper state $i$ $N_{i}$ is expressed by the following equation

$$
I_{i, j}=\frac{h c}{\lambda_{i, j}} A_{i, j} N_{i}=\frac{h c}{\lambda_{i, j}} \frac{g_{i}}{Z} A_{i, j} N \exp \left(-\frac{\varepsilon_{i}}{k_{B} T_{e}}\right)
$$

where $h$ is the Plank constant, $c$ is the speed of light, $\lambda$ is the wavelength, $Z$ is the partition function, and $N$ is the total number of the population. The above equation is deformed as follows

$$
\ln \left(\frac{I_{i, j} \lambda_{i, j}}{A_{i, j} g_{i}}\right)=-\frac{\varepsilon_{i}}{k_{B} T_{e}}+\ln K
$$

where $K$ is the constant. Using transition probability, statistical weight, and measured relative radiation intensity, the relative population for electronic excitation $\left(I_{i, j} \lambda_{i, j} / A_{i, j} g_{i}\right)$ is plotted against the energy level $\varepsilon_{i}$. The line fitting to the plots gives the electron temperature because the gradient of the line is proportional to $-1 / k_{B} T_{e}$ in Eq. (15). This is the procedure of the Boltzmann plot method. Figure 6 shows an example of the Boltzmann plot obtained from the measured spectra in this study. It is found that the plots are scattered and the gradient of the fitted line is negative. This means that the electronic excitation population is much different from the Boltzmann distribution in the energy range under consideration. The present result indicates that the argon plasma induced by the hypersonic shock wave is in thermochemical nonequilibrium. Therefore, the electron temperature cannot be obtained using the Boltzmann plot method in the present case.

\subsection{Evaluation of electron temperature}

Table 4 shows the measured and estimated relative population densities at each position from the shock front. By substituting these population densities into Eq. (8), the 
Table 4. Measured and estimated relative population densities.

\begin{tabular}{|c|c|c|c|}
\hline Position, $\mathrm{mm}$ & $N_{20}$ & $N_{25}$ & $N_{27}$ \\
\hline 25.1 & $9.0 \times 10^{14}$ & $2.4 \times 10^{14}$ & $6.6 \times 10^{14}$ \\
\hline 26.1 & $9.9 \times 10^{14}$ & $2.6 \times 10^{14}$ & $7.2 \times 10^{14}$ \\
\hline 27.1 & $1.0 \times 10^{15}$ & $2.6 \times 10^{14}$ & $6.9 \times 10^{14}$ \\
\hline 28.1 & $1.1 \times 10^{15}$ & $2.8 \times 10^{14}$ & $7.4 \times 10^{14}$ \\
\hline 29.1 & $1.1 \times 10^{15}$ & $3.0 \times 10^{14}$ & $8.1 \times 10^{14}$ \\
\hline 30.1 & $1.0 \times 10^{15}$ & $2.6 \times 10^{14}$ & $6.9 \times 10^{14}$ \\
\hline 31.1 & $9.6 \times 10^{14}$ & $2.5 \times 10^{14}$ & $6.5 \times 10^{14}$ \\
\hline 32.1 & $8.6 \times 10^{14}$ & $2.3 \times 10^{14}$ & $6.3 \times 10^{14}$ \\
\hline 33.1 & $7.6 \times 10^{14}$ & $1.9 \times 10^{14}$ & $5.0 \times 10^{14}$ \\
\hline 34.1 & $6.3 \times 10^{14}$ & $1.6 \times 10^{14}$ & $4.4 \times 10^{14}$ \\
\hline
\end{tabular}

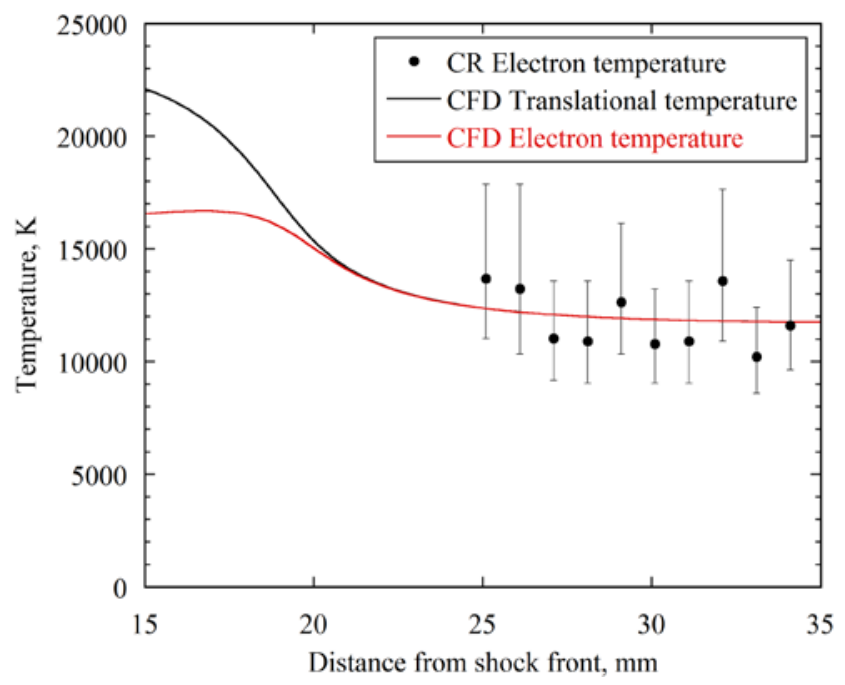

Fig. 7. The spatial distribution of temperatures.

spatial distribution of the electron temperature is obtained as shown in Fig. 7. In the figure, plots are the electron temperature obtained using the CR model. The black and red lines are the translational and electron temperatures obtained by CFD calculation, respectively. The detail of CFD calculation is described in a previous study. ${ }^{[9]}$ The electron temperature obtained using the CR model qualitatively agrees well with the calculated one. However, these values are slightly scattered due to the deviation of the peak intensities in the measured spectra. The error bars are deduced by considering $7 \%$ uncertainty in the population densities, which are estimated from the measured intensities. The calculated temperature profile indicates that the flow region where the CR model is applied is in a thermal equilibrium state. However, our previous study shows that the calculated electron density disagrees with that obtained from the $\mathrm{H}-\beta$ line profile under the same experimental conditions. ${ }^{9)}$ The measured electron density lies between $3.0 \times 10^{21}$ and $4.5 \times 10^{21} \mathrm{~m}^{-3}$, while the calculated one lies between $7.0 \times 10^{21}$ and $2.3 \times 10^{22} \mathrm{~m}^{-3}$, showing the difference by one order of magnitude at most. The disagreement in the electron density is considered to have little effect on the electron temperature evaluation obtained using the CR model. This is because that the plasma is in the ladder-like transition regime even if the electron density differs by one order of magnitude under the condition in this study, as shown in Fig.3.

Fujita et al. ${ }^{18)}$ reported a similar discrepancy of the electron density between measurement and calculation in their shock tube experiment using nitrogen as the test gas. A possible cause of the disagreement in electron density is the precursor phenomena reported in past studies. ${ }^{8,}$ 19-22) This is because precursor phenomena are considered to affect the ionization process and relaxation distance behind a shock wave. Another possible cause is the exclusion of the electronic excitation of argon atoms in this study. Figure 6 shows that the electronic excitation of argon atoms is in a thermal nonequilibrium state. If the electron density is calculated accurately using improved CFD modeling considering the mechanisms mentioned above, the calculated translational and electron temperatures can differ, indicating the flow region examined in this study is in a thermal nonequilibrium state behind the shock wave. However, there is a possibility that the calculated electron temperature disagrees with the electron temperature obtained using the CR model. Therefore, the validity of the electron temperature obtained using the CR model is investigated based on available data in similar studies. Nelson ${ }^{22}$ c conducted a theoretical study of precursor phenomena for shock waves propagating at 6.98 and $7.23 \mathrm{~km} / \mathrm{s}$ in argon gas at a pressure of $1330 \mathrm{~Pa}$. The result shows that the electron temperature equilibrates with the translational temperature immediately behind the shock waves. In the nonequilibrium region, the electron temperatures are about $15000 \mathrm{~K}$ for 6.98 $\mathrm{km} / \mathrm{s}$ and $17000 \mathrm{~K}$ for $7.23 \mathrm{~km} / \mathrm{s}$, respectively. Katsurayama et al. ${ }^{23)}$ investigated precursor photoionization for an extremely strong shock wave propagating at $13.2 \mathrm{~km} / \mathrm{s}$ in argon gas at a pressure of $39 \mathrm{~Pa}$. The calculated electron temperature in the nonequilibrium region is about $25000 \mathrm{~K}$. In addition, this study shows that precursor photoionization has little effect on the value of the calculated electron temperature. The electron temperature obtained using the $\mathrm{CR}$ model is different from these electron temperatures due to the difference in conditions. However, roughly speaking, the order of the electron temperature obtained using the CR model is almost the same as those of the electron temperatures in these studies. So, the agreement of the electron temperature between measurement and calculation is considered to hold if we use an improved CFD modeling.

Although validation of the CR model is not sufficient due to the lack of available data, the present study has indicated a possibility of the simple CR model for determining the electron temperature of nonequilibrium argon plasma induced by a hypersonic shock wave.

\section{Conclusion}


In the present study, optical diagnostics of a nonequilibrium argon plasma induced by a hypersonic shock wave were conducted by applying a simple CR model. The emission spectra of the argon plasma were observed using spectroscopic measurements. In the measured spectrum, line spectra of argon atoms are predominant and clearly seen in the NIR region. Line spectra of argon atoms and ions are observed in the VIS region. However, the influence of contaminated gases is significant. The electronic excitation population was deduced by applying the Boltzmann plot method to the measured line spectra of argon atom. It was found that the electronic excitation population does not follow the Boltzmann distribution, showing that the argon plasma is in a thermal nonequilibrium state. The spatial distribution of the electron temperature was deduced using the CR model and compared with that of calculated one. The result shows that the deduced electron temperature qualitatively agrees well with the calculated one. However, the calculated electron density using CFD disagrees with the measured electron density, indicating that other possible mechanisms should be considered for CFD calculation. Although the validation of the CR model is not sufficient, the present study has indicated the possibility of using a simple CR model for determining the electron temperature of a nonequilbirium argon plasma induced by a hypersonic shock wave. In the future, the applicability of the model will be investigated in other test conditions.

\section{Acknowledgments}

This work was supported by JKA through its promotion funds from KEIRIN RACE.

\section{References}

1) Park, C.: Nonequilibrium Hypersonic Aerothermodynamics, John Wiley \& Sons, New York, 1990.

2) Gnoffo, P. A., Gupta, R. N. and Shinn, J. L.: Conservation Equations and Physical Models for Hypersonic Air Flows in Thermal and Chemical Nonequilibrium, NASA TP-2867, 1989.

3) Gupta, R. N., Yos, J. M., Thompson, R. A., and Lee, K. P.: A Review of Reaction rates and Thermodynamic and Transport Properties for the 11-Species Air Model for Chemical and Thermal Nonequilibrium Calculations to 30000 K, NASA TM-101528, 1989.

4) Park, C.: Review of Chemical-Kinetic Problems of Future NASA Missions, I: Earth Entries, Journal of Thermophysics and Heat Transfer, 7, 3 (1993), pp. 385-398.

5) Yamada, G., Takayanagi, H., Suzuki, T. and Fujita, K.: Shock Layer Radiation Analysis using a Hypervelocity Shock Tube (HVST), Transactions of the Japan Society for Aeronautical and Space Sciences 55, 1 (2012), pp.37-43.

6) Yamada, G., Takayanagi, H., Suzuki, T. and Fujita, K.: Analysis of Shock Layer Radiation from the Vacuum-Ultraviolet to
Near-Infrared Regions, Transactions of the Japan Society for Aeronautical and Space Sciences 55, 1 (2012), pp.60-67.

7) Yamada, G. Takayanagi, H. Suzuki, T. and Fujita, K.: Numerical Analysis on the Nonequilibrium Phenomena of the Electronic Excitation Process behind Hypersonic Shock Waves, Transactions of the Japan Society for Aeronautical and Space Sciences, 56, 2 (2013), pp.63-69.

8) Yamada, G., Ago, S., Kawazoe, H. and Obayashi, S.: Generation mechanism of precursor electrons ahead of a hypersonic shock wave in argon, Journal of fluid science and technology, 9, 5 (2014), JFST0070.

9) Yamada, G., Kawazoe, H. and Obayashi, S.: Electron Density Measurements behind a Hypersonic Shock Wave in Argon, Journal of fluid science and technology, 11, 1 (2016), JFST0005.

10) Yanguas-Gil, A. Cotrino, J. and Gonzalez-Elipe, A. R.: Influence of the excited states on the electron-energy distribution function in low-pressure microwave argon plasmas, Physical review E 72 (2005), pp.1-9.

11) Vlcek, J.: Acollisional-radiative model applicable to argon discharges over a wide range of conditions. I: Formulation and basic data, Journal of physics D: Applied physics, 22 (1989), pp.623-631.

12) Kimura, A. Kobayashi, H. Nishida, M. and Valentin, P.: Calculation of collisional and radiative transition probabilities between excited argon levels, Journal of Quantitative Spectroscopy Radiative transfer, 34 (1985), pp.189-215.

13) Bogaerts, A. Gijbels, R. and Vlcek, J.: Collisional-radiative model for an argon glow discharge, Journal of applied physics, 84 (1998), pp.121-136.

14) Bultel, A. Ootegem, B. Bourdon, A. and Vervisch, P.: Influence of $\mathrm{Ar}^{+}$in an argon collisional-radiative model, Physical review E, 65, 2 (2002), pp.1-15.

15) Kano, K. Suzuki, M. and Akatuka, H.: Spectroscopic measurement of electron temperature and density in argon plasmas based on collisional-radiative model, Plasma source science technology, 9 (2000), pp.314-322.

16) Yamada, G. Ago, S. Kubo, Y. Matsuno, T. and Kawazoe, H.: Development of a Shock Tube Facility for Nonequiliibrium Radiation Studies in Mars Entry Flight Conditions, Journal of Space Engineering, 6 (2013), pp.28-43.

17) Fujimoto, T.: Plasma Spectroscopy, Oxford University Press, 2004.

18) Fujita, K. Sato, S. and Abe, T.: Electron Density Measurements Behind Strong Shock Waves by H- $\beta$ Profile Matching, Journal of Thermophysics and Heat Transfer, 17, 2 (2003), pp.210-216.

19) Weymann, D. H.: Precursors Ahead of Shock Waves: I. Electron Diffusion, The physics of fluids, 12, 6 (1969), pp.1193-1199.

20) Holmes, B. L. Weymann, D. H.: Precursors Ahead of Shock Waves: II. Photoionization, The physics of fluids, 12, 6 (1969), pp.1200-1210

21) Foley, H. W. Clarke, H. J.: Shock Waves structured by nonequilibrium ionizing and thermal phenomena, The physics of fluids, 16, 3 (1973), pp.375-383.

22) Nelson, F. H.: Nonequilibrium structure of argon shock waves, The physics of fluids, 16, 12 (1973), pp.2132-2142.

23) Katsurayama, H., Matsuda, A. and Abe, T.: Preliminary investigation of thermochemical nonequikibrium behind a strong shock wave with precursor photoionization in argon, 39 $9^{\text {th }}$ AIAA Thermophysics Conference, AIAA-2007-4552, 2007. 